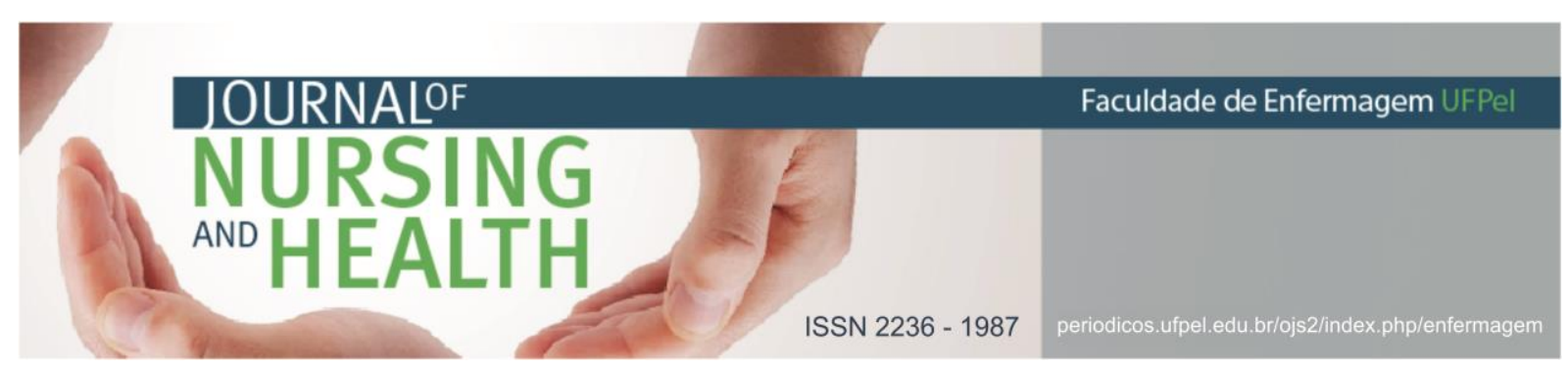

RELATO DE EXPERIÊNCIA

\title{
A construção do Jornal do Centro de Atenção Psicossocial II: desenvolvendo habilidades específicas e relacionais
}

\author{
Editing the Center for Psychosocial Care: developing hard and soft skills
}

\section{La construcción del "Periódico CAPS": desarrollando habilidades específicas y relacionales}

Castan, Juliana Unis ${ }^{1}$; Brentano, Vivian²; Ipuchima, Jaqueline Ramires ${ }^{3}$; Moraes, Helena Abadie $^{4}$

\section{RESUMO}

Objetivo: demonstrar as possibilidades de uma oficina multiprofissional no desenvolvimento de habilidades específicas e de relacionamento. Métodos: relato de experiência descrevendo a construção do Jornal do Centro de Atenção Psicossocial (CAPS). Resultados: empoderamento através do aprendizado de ferramentas como computador e internet; desenvolvimento de capacidades relacionais, como liderança, autonomia, exposição de ideias e trabalho em equipe. Considerações finais: destaca-se o potencial de uma atividade organizadora, cujo produto final é um material impresso, para o crescimento e desenvolvimento de competências e habilidades específicas e relacionais, aspectos importantes para a efetiva reinserção social.

Descritores: Reabilitação; Habilidades sociais; Aptidão; Promoção em saúde; Transtornos mentais.

\begin{abstract}
Objective: to demonstrate the possibilities of a multidisciplinary workshop on developing hard and soft skills to improve rehabilitation. Methods: experience's description of conducting a working group aimed to writing and editing a newspaper. Through such specific task, different hard and soft skills are developed. Hard skills are specific abilities, such as using the computer and the internet, whereas soft skills are social skills, such as how to relate to others and negotiate. Results: empowerment of the clients by enhancing abilities with tools like the computer and the internet; Development of relational capacities, such leadership and group working. Final considerations: this multidisciplinary therapeutic workshop is useful to enhance clients' work and social skills and improve their rehabilitation process.
\end{abstract}

Descriptors: Rehabilitation; Social skills; Aptitude; Health promotion; Mental disorders.

\footnotetext{
1 Psicóloga. Mestre em Counseling and Personnel Services. Psicóloga no Hospital de Clínicas de Porto Alegre, Porto Alegre, RS, Brasil. Email: jcastan@hcpa.edu.br http://orcid.org/0000-0002-6946-0537

2 Psicóloga. Especialista em Saúde Mental. Psicóloga Clínica na Comunidade Terapêutica Winnicott. Porto Alegre, Rio Grande do Sul. Email: vivianbrentano@gmail.com http://orcid.org/0000-0002-8869-8751

${ }^{3}$ Enfermeira. Especialista em Saúde Mental. Enfermeira na Irmandade Santa Casa de Misericórdia. Porto Alegre, Rio Grande do Sul, Brasil. Email: jaqueramires@hotmail.com http://orcid.org 0000-0002-7446-0566

${ }^{4}$ Nutricionista. Especialista em Saúde Mental. Mestranda na Universidade Federal do Rio Grande do Sul. Porto Alegre, Rio Grande do Sul, Brasil. Email: hlnmoraes@gmail.com http://orcid.org/0000-0001-7759-3308
} 


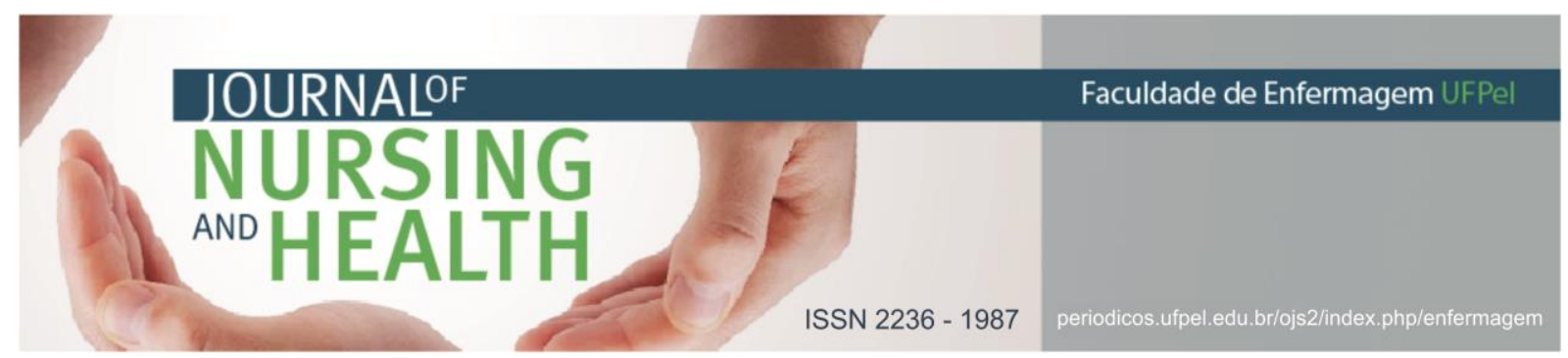

RESUMEN

Objetivo: demonstrar las posibilidades de un taller multiprofesional en el desarrollo de habilidades específicas y relacionales. Métodos: un relato describiendo la construcción del Periódico, pues con una tarea concreta y dirigida es posible trabajar habilidades específicas y de relaciones. Resultados: el empoderamiento de herramientas como la computadora y el internet; el desarrollo de capacidades relacionales, como liderazgo, autonomía, exposición de ideas y trabajo en equipo. Consideraciones finales: se destaca el potencial de una actividad organizadora, en la que el producto final es un material impreso, para el crecimiento y el desarrollo de competencias, habilidades específicas y de relaciones, aspectos importantes para la efectiva reinserción social.

Descriptores: Rehabilitación; Habilidades sociales; Aptitud; Promoción de la salud; Trastornos mentales.

\section{INTRODUÇÃO}

Os Centro de Atenção Psicossocial (CAPS) configuram-se como serviços comunitários, ambulatorial e regionalizado, assumindo o papel de articulador da rede de saúde, aproximando a saúde coletiva e a saúde mental, constituindo assim um campo interdisciplinar de saberes e práticas. ${ }^{1-2}$ Tem a característica de ser um tratamento intermediário, entre o atendimento ambulatorial e a internação psiquiátrica em hospitais gerais.

O CAPS é um local de referência e de tratamento para pessoas que possuem transtornos mentais graves $\mathrm{e}$ persistentes, tendo por objetivo a reinserção social, buscando o convívio com pares, familiares e demais membros da sociedade, bem como a ocupação cidadã dos espaços sociais. ${ }^{3-}$ 4 Visa a reabilitação psicossocial, conseguida através do fortalecimento de potencialidades e do desenvolvimento de formas de lidar com efeitos negativos e cronificadores da doença. A reabilitação é percebida através do enfrentamento das dificuldades e limitações e da melhora no autocuidado. ${ }^{5}$ Dessa forma, mais que um ambiente que proporcione momentos agradáveis, os CAPS devem servir como uma possibilidade de mudança social, assumindo papel terapêutico no tratamento do usuário, visando sua inserção e contribuição à sociedade. ${ }^{6}$

Para efetivação do tratamento, é primordial que usuário e família participem do processo. Para tanto, os CAPS utilizam-se do Projeto Terapêutico Singular (PTS). Através da participação do usuário e sua família, o PTS estimula a construção de autonomia, considerando as necessidades do indivíduo e o contexto em que está inserido. A elaboração do PTS acontece por meio da atuação do usuário/família e do profissionalreferência, junto à equipe, discutindo as necessidades dos usuários e reavaliando as intervenções, considerando serviços e instituições da rede de saúde e do contexto. ${ }^{7-9}$ No PTS são descritos o plano e os objetivos do tratamento, assim como estratégias para alcançá-los e resultados esperados. Como estratégias para buscar o objetivo final - a reinserção e participação social -, os planos valemse de tratamento medicamentoso, consultas individuais, atendimentos 


\section{JOURNALOF \\ NURSING \\ ANOHEALTH}

em forma de grupo e oficinas terapêuticas, além de acompanhamento na rede e visitas domiciliares.

As oficinas terapêuticas surgiram com o intuito de propiciar uma maior facilidade nas relações sociais dos usuários dos serviços de saúde mental com suas famílias e com a sociedade. ${ }^{10}$ Através das oficinas, é possível desconstruir saberes prévios, criar alternativas e compartilhar vivências e sofrimentos. ${ }^{11}$ Dessa forma, usuários aprendem a aceitar diferenças, particularidades e possibilidades de cada sujeito. 0 reflexo deste trabalho é observado na melhora da autoestima, aspecto relacionado com taxas de sucesso na reabilitação de indivíduos com transtorno mental. ${ }^{12}$

Assim, quando a oficina consegue produzir subjetividades, catalisar afetos, explorar pensamentos e sentimentos, ela adquire valor na reabilitação, possibilitando ao usuário trabalhar e descobrir suas potencialidades para conquistar espaços sociais. A doença mental pode adquirir novos sentidos e significados dentro da trajetória de vida daquele indivíduo. Dessa forma, as oficinas cumprem a finalidade de reabilitação psicossocial ao promoverem ambientes de (re) construção de papéis sociais e possibilitarem trocas com os espaços sociais. ${ }^{13}$

A técnica de grupo operativo, desenvolvida por Pichon-Riviere, é um importante recurso para o trabalho em oficinas. Esta técnica preconiza que o grupo reúna-se em torno de um objetivo comum, também chamado de tarefa explícita. Entretanto, por trás da tarefa explícita, há a tarefa
ISSN 2236 - 1987

implícita, que se refere à possibilidade de crescimento e aprendizagem, através da quebra de estereótipos e padrões rígidos de funcionamento. ${ }^{14}$ Mais importante que o produto final é o processo de aquisição de conhecimento e mudança, tendo este um caráter terapêutico. É o conjunto de ideias, afetos e experiências que os membros do grupo trazem na bagagem, modificam e desenvolvem no fazer grupal que constitui a tarefa implícita.

Considerando reinserção e reabilitação como o objetivo final do tratamento em CAPS, percebeu-se a necessidade de desenvolver uma oficina terapêutica que, através da técnica de grupo operativo, preconizasse questões de autonomia e desenvolvimento da identidade, ao mesmo tempo em que trabalhasse habilidades específicas, como a escrita e o uso do computador. Assim, foi criado o projeto da oficina do Jornal do CAPS. Este artigo visa descrever a elaboração e desenvolvimento de uma oficina terapêutica que tem por objetivo final produzir um material gráfico na forma de jornal.

\section{MATERIAIS E MÉTODOS}

Este artigo busca descrever a experiência do desenvolvimento e condução da oficina do Jornal, no período de um ano, realizado em um CAPS da região sul do Brasil. 0 trabalho foi coordenado pela psicóloga contratada do serviço e pelas residentes da Residência Integrada Multiprofissional em Saúde - ênfase em Saúde Mental, nas áreas de Enfermagem, Nutrição e Psicologia. 


\section{ISSN 2236 - 1987}

O grupo da oficina do Jornal é composto por cerca de 20 usuários. É um grupo aberto, com encontros semanais de duas horas de duração. A indicação para participação no grupo é feita através do Projeto Terapêutico Singular, sendo respeitada a vontade e necessidade do usuário em desenvolver habilidades específicas e relacionais, além de requisitos básicos como não estar em episódio psicótico agudo que impeça o envolvimento nas atividades e interessar-se por tarefas que envolvam o escrever, pesquisar $e$ discutir.

Constitui-se um grupo bastante heterogêneo no que diz respeito à idade, sexo, patologia, interesses e capacidades, tendo desde usuários funcionalmente analfabetos até usuários com Ensino Médio Completo. Da mesma forma, há indivíduos entre 18 e 70 anos de idade, com as mais diversas experiências e objetivos de vida. Como um fator comum aos membros do grupo, além do interesse pelo tema e a necessidade de desenvolver estas habilidades, tem-se a experiência do transtorno mental e a dificuldade de reinserção na sociedade, visto que é a esta população à que o CAPS se destina.

Para a operacionalização do grupo, os usuários são divididos em quatro equipes que funcionam como grupos de trabalho. Cada equipe tem como facilitador um residente multiprofissional. 0 papel dos facilitadores é auxiliar os usuários no desenvolvimento de habilidades específicas, como usar o computador e pesquisar em plataforma de buscas da internet, e relacionais, mediando relações e servindo como modelo para os usuários. Esses profissionais em formação buscam, durante todo o processo, estimular a autonomia e independência dos usuários, incentivando-os, inclusive, a assumir o papel de líder da equipe. A atividade é coordenada e supervisionada pela psicóloga contratada da instituição.

No início de cada encontro, é retomado em grande grupo as seções do Jornal já desenvolvidas e as faltantes. Cada equipe escolhe em que seção do Jornal trabalhará naquele dia. Espera-se que as equipes possam circular entre as seções, assumindo diferentes responsabilidades em cada edição do Jornal, visto que as seções exigem habilidades e competências diferentes. Os usuários ficam responsáveis pela execução das tarefas, cabendo aos facilitadores estimular a participação e autonomia.

Espera-se que, progressivamente, cada equipe possa se autogerir, com usuários estimulando e ensinando uns aos outros, tornando a troca de conhecimentos e experiências um fator de reconhecimento de um lugar social. Ao final de cada encontro, as equipes se reúnem para apresentar sua produção daquele dia e iniciar o planejamento do próximo encontro. Dessa forma, todos acompanham o andamento geral da atividade.

A diagramação e planejamento visual também fazem parte do processo, sendo realizados por uma das equipes e permitindo o aprimoramento de habilidades relacionadas ao computador, além das relacionais. Quando a diagramação está finalizada, é impressa uma cópia piloto, que passa pela revisão dos usuários antes da impressão final de 100 exemplares, 


\section{NURSING \\ AND}

distribuídos entre os usuários, profissionais e comunidade. Essa impressão é realizada na gráfica do hospital ao qual o CAPS é vinculado.

Para o desenvolvimento desta atividade, o CAPS conta com recursos pessoais, psicóloga contratada e residentes multiprofissionais em saúde, e estrutura física com salas e computadores com acesso à internet. Além disso, o hospital ao qual o CAPS é vinculado oferece apoio através da sua gráfica, onde são impressos os exemplares de cada edição. A edição é bimestral, tendo as seguintes seções: Gastronomia; Dica Útil; Filme; Música; Poema; Curiosidades, Datas Comemorativas, Aniversariantes; Cultura Através da Arte; Notícias da Comunidade; Entrevistas e Eventos.

\section{RESULTADOS E DISCUSSÃO}

No formato descrito acima, foram realizadas quatro edições bimestrais do Jornal, com média de 15 a 20 participantes por encontro. Ressaltase a heterogeneidade do grupo, composto por indivíduos com diferentes características de personalidades, funcionamento cognitivo, patologias e interesses. Essa multiplicidade, apesar de desafiadora para a equipe, mostra-se um fator de enriquecimento. Os participantes têm a chance de aprender a lidar com as diferenças, reconhecer-se, identificar facilidades e dificuldades e aprender a negociá-las a fim de cumprir com o objetivo da elaboração do Jornal. Assim, em meio a diferenças e semelhanças, encontros e desencontros, se dá a construção da identidade, aspecto essencial para a reabilitação. ${ }^{12}$
ISSN 2236 - 1987

Por ser um grupo aberto, há usuários ingressando ao longo da atividade. Isso, apesar de exigir paradas no andamento do processo, permite que este seja revisto e repensado, tanto pelos profissionais como pelos usuários, imprimindo um caráter dinâmico e flexível à atividade. Além disso, esta característica permite que os usuários desenvolvam habilidades relacionais, como receber um colega, explicar o trabalho, acolhêlo. Cabe ressaltar que o ingresso de pessoas em equipe de trabalho é uma situação frequente em atividades laborais, fazendo com que esta oficina funcione como um laboratório de preparação para o mercado de trabalho.

Durante o processo, pode-se observar o desenvolvimento das habilidades específicas, em especial relacionadas ao computador e à internet, acompanhadas por sentimento de orgulho e de superação. Durante a oficina, usuários comentam como conseguem se relacionar melhor com filhos e netos, mostrando o valor destas ferramentas no convívio social nos dias atuais. 0 impacto destes sentimentos e vivências na autoestima é visível e participantes dão voz a isso quando comentam que agora fazem tarefas que achavam que seriam incapazes. Além disso, o surgimento do desejo e interesses profissionais parece permeado por esta vivência, como por exemplo, quando uma usuária refere que deseja trabalhar em serviços administrativos.

Com relação às habilidades relacionais, destacam-se as possibilidades do trabalho em pequenos grupos e a assunção de 


\section{JOURNAIOF \\ NURSING \\ ANO HEALTH}

ISSN 2236 - 1987

responsabilidades. Usuários percebem sua importância na equipe ao verem que a finalização de uma matéria depende de sua participação. Dessa forma, questões de responsabilidade, autogestão e iniciativa são trabalhadas. Através da resolução de conflitos, do foco e cooperação em torno de um objetivo comum, usuários descobrem novas formas de se relacionar uns com os outros e com a tarefa. Com o desenvolvimento do trabalho, percebeu-se que, cada vez menos, os facilitadores tinham que assumir uma postura de cobrança ou de delegar tarefas. Pelo contrário, os usuários cobravam e estimulavam uns aos outros, permitindo 0 desenvolvimento de capacidades relacionadas à autonomia, independência, responsabilidade e comprometimento.

Cada equipe funciona como um microcosmo, onde o usuário pode, de forma não necessariamente consciente, treinar habilidades adquiridas, assumir responsabilidades, trocar papéis. Assim, pode-se observar como participantes conseguem vencer o receio e medo de se expor, que, por vezes, tinha efeito paralisador. A troca de papéis permite a descoberta de novos interesses e de outras facetas da identidade ${ }^{13,14}$. De forma indireta e constante, usuários treinam e aprendem, através do feedback de seus pares, a expor sua opinião, escutar aos outros, negociar, divergir e acordar. Essas são habilidades essenciais para a convivência em sociedade e participação em espaços coletivos.

As seções de Música e Poema permitem o uso da criatividade, uma vez que são feitas paródias de músicas conhecidas e selecionadas pelos usuários, assim como o poema é uma criação dos mesmos. Pensamento crítico é trabalhado especialmente através da seção do Filme, em que os usuários escrevem uma resenha crítica de um filme selecionado por eles. Notícias da Comunidade permite que os usuários deem voz a sua realidade, descrevendo como a veem, possibilitando que novos sentidos sejam dados à experiência vivida. A apreensão e ressignificação da realidade, tanto interna quanto externa, tem papel importante no processo de reabilitação e reinserção social. ${ }^{13}$

As seções de entrevista, eventos do CAPS e notícias da comunidade mostram-se com um potencial extra, pois, além de permitir o desenvolvimento das habilidades citadas, refletem a realidade dos usuários, dando visibilidade ao mundo como percebem e sentem. Ao retratarem questões de suas realidades, muitas vezes permeadas de vulnerabilidades e violência, estas podem ser pensadas e adquirirem novos significados. ${ }^{13}$ Além disso, e por outro lado, é possível observar o sentimento de orgulho e pertença. A escolha das fotos para a seção de eventos, assim como aparecer em um veículo de comunicação, mostram-se fontes importantes de prazer e reconhecimento.

Por fim, destaca-se o sentimento de orgulho em produzir. Sabe-se do valor do trabalho como um local social, que dá legitimidade e poder ao indivíduo. Ao produzirem um Jornal, ao verem impressas suas criações e ideias, 


\section{JOURNALOF \\ NURSING \\ AND HEALTH}

ISSN 2236 - 1987

esse sentimento torna-se evidente. Esse orgulho e contentamento podem ser exemplificados pela solicitação de exemplares do Jornal para levarem para suas famílias, outros serviços, como Unidades Básicas de Saúde e demais locais da comunidade. 0 Jornal, aqui, aparece como uma possibilidade de visibilidade, de ser visto e reconhecido na sua comunidade e, consequentemente, na sociedade. Com o desenvolvimento da autoestima e da identidade pessoal e social, usuários se fortalecem, aumentando condições para gerir suas vidas e realizar escolhas. ${ }^{5}$

\section{CONSIDERAÇÕES FINAIS}

Percebe-se o potencial de uma atividade dirigida e concreta, que tem como resultado um produto final impresso, para o crescimento e desenvolvimento de competências e habilidades específicas e relacionais, aspectos importantes para a efetiva reinserção social, tanto no âmbito profissional como social. A oficina da criação e editoração do Jornal mostrou-se um espaço para construção de autonomia, cidadania, construção coletiva e protagonismo. Usuários descobrem novas capacidades e facetas de sua identidade, permitindo e incentivando autodescoberta e descoberta de uns aos outros.

Crescimento e aprendizagem, base do grupo operativo, permitem quebra de estereótipos e mudança do status quo. Através do desenvolvimento da identidade e do autoconhecimento, usuários podem assumir um papel ativo frente a seu processo de saúde-doença, consolidando, na prática, questões de

protagonismo. Ao melhorarem
autoestima e desenvolverem conhecimento de mundo, fortificam-se para tomarem decisões e gerenciarem suas vidas. Assim, ressalta-se a importância de oficinas em que os usuários são protagonistas, saindo de uma posição passiva para assumirem responsabilidades na condução, primeiro de uma tarefa, mas também de seu tratamento e projeto de vida.

Cabe ressaltar que muito dos resultados de um trabalho em grupo se dão a longo prazo. Mudanças na autoestima, assim como questões na identidade, tendem a ser sutis e construídas ao longo do tempo. 0 CAPS, por ser um serviço regionalizado, de média a longa permanência, permite que estas mudanças a posteriori sejam observadas e testemunhadas pelos profissionais envolvidos no processo, trazendo satisfação e reconhecimento também à equipe envolvida.

\section{REFERÊNCIAS}

1. Jorge MSB, Diniz AM, Lima LL, Penha JC. Apoio matricial, projeto terapêutico singular e produção do cuidado em saúde mental. Texto contexto enferm. 2015;24(1):112-20.

2. Nascimento GCM, Scorsolini-Comin F, Peres RS. Saúde Mental no Sistema Único de Saúde: mapeamento das contribuições dos Centros de Atenção Psicossocial. SMAD, Rev eletrônica saúde mental alcool drog. 2013;9(2):95-102.

3. Ministério da Saúde (BR). Saúde mental no SUS: os centros de atenção psicossocial. Brasília; 2004. 
4. Lobato DSP, Sato LE, Brito SR. Oficina terapêutica de inclusão digital como instrumento auxiliador no tratamento de pacientes com transtorno mental do Centro de Apoio Psicossocial do Pará. Extramuros. 2015;3(3):39-50.

5. Jorge MSB, Pinto DM, Quinderé PHD, Pinto AGA, Sousa FSP, Cavalcante CM. Promoção da Saúde MentalTecnologias do Cuidado: vínculo, acolhimento, co-responsabilização e autonomia. Ciênc saúde colet. 2011;16(7):3051-60.

6. Nascimento A, Gaio DM. Possibilidades de recursos terapêuticos para pacientes psicóticos. Cadernos da escola de saude. 2016;1(15):81-95.

7. Ministério da Saúde (BR). Secretaria de Atenção à Saúde. Núcleo Técnico da Política Nacional de Humanização. Clínica ampliada, equipe de referência e projeto terapêutico singular. $2^{\mathrm{a}}$ ed. Brasília; 2008.

8. Carvalho LGP, Moreira MDS, Rézio LA, Teixeira NZF. A construção de um Projeto Terapêutico Singular com usuário e família: potencialidades e limitações. Mundo saúde. 2012;36(3):521-5.

9. Rodrigues J, Deschamps ALP. Política de Saúde Mental e Projeto terapêutico Singular. Cadernos brasileiros de saude mental. 2016;8(17):79-92.

10. Scholz DCS, Corrêa MM, Duarte MLC, Torres OM, Balk RS, Strack EM. A construção do projeto terapêutico de um CAPS no sul do Brasil. Rev contexto saude. 2014;14(27):65-9.

11. Martinhago F, Oliveira WF. A prática profissional nos Centros de
ISSN 2236 - 1987

Atenção Psicossocial II (CAPS II), na perspectiva dos profissionais de saúde mental de Santa Catarina. Saúde debate. 2012;36(95):583-94.

12. Torrey WC, Mueser KT, McHugo GH, Drake RE. Self-esteem as an outcome measure in studies of vocational rehabilitation for adults with severe mental illness. Psychiatr ser. 2000;51(2):229-33.

13. Guerra AMC. Oficinas em saúde mental: um percurso de uma história, fundamentos de uma prática. In: Costa CM, Figueiredo, AC. Oficinas terapêuticas em saúde mental: sujeito, produção e cidadania. Rio de Janeiro: Contra Capa; 2004.

14. Pereira TTSO. Pichon Riviere, a dialética e os grupos operativos: implicações para pesquisa e intervenção. Rev SPAGESP. 2013;14(1):21-9.

Data de submissão: 21/04/2017

Data de aceite: 01/11/2017

Data de publicação: 26/12/2017 